\title{
Bio-Stimulation of Ureolytic Bacteria for Calcite Precipitation in Desert Soils
}

\author{
HADAS RAVEH-AMIT ${ }^{1}$ AND MICHAEL TSESARSKY ${ }^{2}$
}

${ }^{1}$ Nuclear Research Centre Negev

${ }^{2}$ Ben-Gurion University of the Negev

Presenting Author: hadas.raveh@gmail.com

Microbial-induced calcite precipitation (MICP) is a soil amelioration technique aimed to mitigate different environmental and engineering concerns, including desertification, soil erosion and soil liquefaction, among others. Hydrolysis of urea, catalyzed by the microbial enzyme urease, is considered the most efficient microbial pathway for MICP. Bio-stimulated MICP relies on the enhancement of indigenous urea-hydrolyzing bacteria by providing an appropriate enrichment and precipitation media, as opposed to bio-augmentation, which requires introducing large volumes of exogenous bacterial cultures into the treated soil along with growth and precipitation medium. Bio-stimulated MICP in desert soils is challenging as the total carbon content and the bacterial abundance are considerably low. In this study, we examined the bio-stimulation potential in soils from the Negev Desert, Israel. Successful biostimulation of ureolytic bacteria was achieved by incubating soil samples in urea and enrichment media and testing for urea hydrolysis and $\mathrm{pH}$ increase. Following stimulation, calcium carbonate precipitation was induced by spiking stimulated bacteria in precipitation $(\mathrm{CaCl} 2$ enriched) media. Increased concentrations of energy (carbon) source in the stimulation media, resulted in higher bio-stimulation rates, reaching its maximal levels within 3 to 6 days. The effectiveness of bio-stimulation was tested and compared between different locations and at different depths. Bio-stimulation was most effective at the topsoil, whereas significantly reduced rates were obtained from samples collected from the subsurface, up to $1 \mathrm{~m}$ below the ground. Bacterial abundance and diversity before and following bio-stimulation were examined and provided information on variability in bio-stimulation rates along the tested sites. Altogether, our research demonstrates that biostimulated MICP is feasible in the low carbon, low mineral soils of the northern Negev Desert in Israel and provides new insights on its effectiveness. 